\title{
Exercise and Motor Training in People with Parkinson's Disease: A Systematic Review of Participant Characteristics, Intervention Delivery, Retention Rates, Adherence, and Adverse Events in Clinical Trials
}

\author{
Natalie E. Allen, ${ }^{1}$ Catherine Sherrington, ${ }^{2}$ Gayanthi D. Suriyarachchi, ${ }^{1}$ \\ Serene S. Paul, ${ }^{1}$ Jooeun Song, ${ }^{1}$ and Colleen G. Canning ${ }^{1}$ \\ ${ }^{1}$ Clinical and Rehabilitation Research Group, Faculty of Health Sciences, The University of Sydney, P.O. Box 170, Lidcombe, \\ Sydney, NSW 1825, Australia \\ ${ }^{2}$ Musculoskeletal Division, The George Institute for Global Health, The University of Sydney, Sydney, NSW 2050, Australia
}

Correspondence should be addressed to Natalie E. Allen, natalie.allen@sydney.edu.au

Received 13 July 2011; Accepted 18 August 2011

Academic Editor: Gammon M. Earhart

Copyright (c) 2012 Natalie E. Allen et al. This is an open access article distributed under the Creative Commons Attribution License, which permits unrestricted use, distribution, and reproduction in any medium, provided the original work is properly cited.

\begin{abstract}
There is research evidence that exercise and motor training are beneficial for people with Parkinson's disease (PD), and clinicians seek to implement optimal programs. This paper summarizes important factors about the nature and reporting of randomized controlled trials of exercise and/or motor training for people with PD which are likely to influence the translation of research into clinical practice. Searches identified 53 relevant trials with 90 interventions conducted for an average duration of 8.3 (SD 4.2) weeks. Most interventions were fully supervised (74\%) and conducted at a facility (79\%). Retention rates were high with $69 \%$ of interventions retaining $\geq 85 \%$ of their participants; however adherence was infrequently reported, and $72 \%$ of trials did not report adverse events. Overall, the labor-intensive nature of most interventions tested in these trials and the sparse reporting of adherence and adverse events are likely to pose difficulties for therapists attempting to balance benefits and costs when selecting protocols that translate to sustainable clinical practice for people with PD.
\end{abstract}

\section{Introduction}

In recent years there have been an increasing number of randomized controlled trials assessing the effects of exercise and/or motor training in people with Parkinson's disease (PD). Overall, these trials support exercise and motor training as beneficial in improving walking, balance, muscle strength, and the performance of functional tasks in people with mild-to-moderate PD [1-11]. In order for findings from this research to be of general benefit to people with PD, therapists need to be able to translate the protocols used in the research into clinical practice [12].

Evidence-based practice aims to incorporate and apply high-quality clinical research findings in clinical policy and practice $[13,14]$. However, this can be a challenging task as health practitioners may find it difficult to assess, interpret, and implement research evidence [13]. While evidence about beneficial outcomes is paramount in therapists' decisions to implement a particular intervention, there are other factors that affect how the overall impact of the intervention is interpreted and its potential for widespread clinical application [13-17]. For example, therapists need to consider how the characteristics of participants included in a trial may affect their decision regarding the applicability of the trial intervention with their patients [14]. It is likely that the way in which the intervention was applied in terms of its duration, level of supervision, delivery (i.e., individual versus group), and location (e.g., facilities and equipment required) will influence therapists' decisions to implement that intervention. A research protocol that has been shown to be effective may not be implemented by therapists if they cannot provide adequate supervision over the required time frame or they do 
not have access to necessary facilities or equipment. Finally, information regarding retention, adherence, and adverse events is required so that therapists and patients can weigh up the effectiveness of the intervention against its acceptability and any risks associated with implementation [14].

Therefore, in order to examine the information available to guide the translation of research into clinical practice, we searched randomized controlled trials of exercise and/or motor training for people with PD to determine the

(1) disease severity and cognitive status of the included participants,

(2) duration, supervision, delivery, and location of the interventions,

(3) rates of retention, adherence, and adverse events.

\section{Methods}

2.1. Data Sources and Searches. Randomized controlled trials of exercise and/or motor training for people with PD were identified via database searches of MEDLINE, EMBASE, AMED, PsycINFO, the Cochrane Central Register of Controlled Trials, and CINAHL. The initial search was conducted in 2009, with a subsequent search conducted over 5 days from the 7th of April, 2011. The electronic search strategy used has been previously reported [2]. The Physiotherapy Evidence Database (PEDro; http://www.pedro.org.au/) was also searched, and the reference lists of previously published systematic reviews $[4,5,8,9,18-30]$ were checked for any trials not identified with the database search.

2.2. Study Selection. Trials included were published randomized (or quasi randomized, i.e., not truly random but intended to produce similar groups, such as allocation by odd and even birth dates [31]) controlled trials of people with $\mathrm{PD}$ where at least one of the interventions was an ongoing program of exercise and/or motor training. All forms of exercise (e.g., aerobic, strength, and treadmill walking) and motor training (e.g., cueing and movement strategy training) were included. Whole-body vibration was not considered to be exercise or motor training. Trials were excluded if the intervention was multidisciplinary or was primarily occupational therapy.

The eligibility of trials was determined in a two-stage process. Firstly, all trial titles and abstracts were screened independently by two investigators (N. E. Allen and G. D. Suriyarachchi). Trials were excluded if it was clear that they did not meet the inclusion criteria. Secondly, the full article was obtained for the remaining trials and each trial was assessed independently by at least two investigators (N. E. Allen, C. G. Canning or J. Song), using a standardized form containing the details of the inclusion criteria. Care was taken to identify trials that had been reported in more than one journal article. Where this occurred, the multiple articles were counted as one trial and all articles were used to collect data for that trial.

2.3. Data Extraction. A data collection form was developed, tested on five randomly selected trials and then modified accordingly. All investigators were involved in data extraction, and all data was double-checked by an investigator not involved in its initial extraction (N. E. Allen or J. Song). Discrepancies were resolved by discussion.

Information extracted from each trial included a description of participants (including cognitive status), details of the exercise and motor training program and how it was administered, as well as details regarding retention rates, adherence to the intervention, and monitoring and reporting of adverse events. Retention was defined as the number of participants who completed the trial (i.e., undertook the first or only post-intervention assessment excluding further follow-up assessments) expressed as a percentage of the number of participants who began the trial. Adherence was defined as the number of intervention sessions attended by participants expressed as a percentage of the number of intervention sessions prescribed [15].

\section{Results}

Searching identified 3,539 records, of which 53 trials involving 1,940 participants were found to be eligible for inclusion in the paper (Figure 1) [32]. There were no disagreements between reviewers regarding the inclusion of any articles. The characteristics of the included trials $[1,3,6,7,10,11,33-85]$ are summarised in Table 1.

3.1. Participant Characteristics. Forty (75\%) of the reviewed trials included participants with mild-to-moderate PD (i.e., equivalent to Hoehn and Yahr stage I to III [86]). Seven trials $(13 \%)$ included participants with mild-to-moderately severe PD (i.e., Hoehn and Yahr stage I to IV), while four trials $(8 \%)$ included only participants with mild PD and two trials $(4 \%)$ included only participants with moderate PD (Table 1). Most trials stipulated the cognitive status of included participants. Twenty-nine trials (55\%) used the Mini-Mental State Examination [87] to screen potential participants' cognitive abilities, with the minimum score for inclusion varying between 20 and 28 out of the maximum of $30[1,3,6,7,10,11,34,36,39,41-43,45,47-50,56$, $58,60,63-65,69,71,76-78,80,85]$. One trial $(2 \%)[70]$ specified that participants required at least moderate scores on the Neurobehavioural Cognitive Status Examination [88]. Thirteen trials $(25 \%)$ made a statement to the effect that included participants had no dementia and/or reasonable cognition [35, 46, 51, 52, 57, 59, 62, 66, 67, 79, 81, 83, 84]. Ten trials (19\%) did not give a clear indication of the participants' cognitive abilities $[33,37,40,53,55,68,72,74,75,82]$.

\subsection{Exercise and/or Motor Training Program Characteristics.} In the 53 trials, there were 90 intervention groups that involved exercise and/or motor training (including two intervention groups for the cross-over trials where one intervention was a control $[11,42,47])$ (Table 1). Average intervention duration was 8.3 weeks $(S D=4.2$, range $=2$ to 26 weeks), with 37 trials (70\%) conducting an intervention of 10 weeks or less. The total number of hours of intervention was not clearly reported in all studies (see Table 1); however, from the available data, an average of approximately 20 hours (SD 


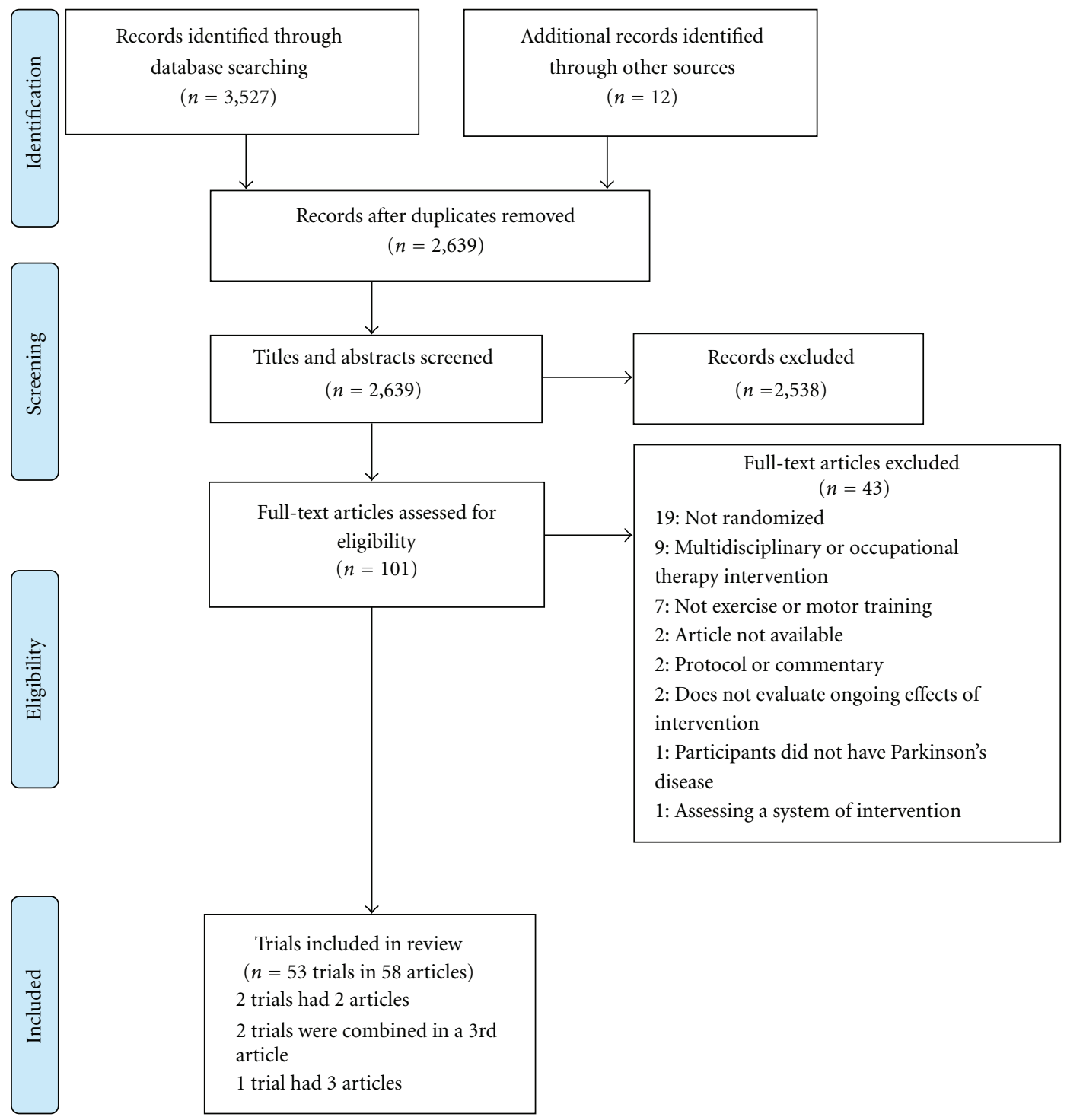

FIgURE 1: PRISMA flow diagram [32] showing flow of information through the review.

approximately 11 , range $=4$ to 65 hours) appears broadly representative of the included trials. Sixty-seven of the 90 intervention groups $(74 \%)$ involved full supervision of exercise and/or motor training. Participants in $18(27 \%)$ of the fully supervised intervention groups received one-onone supervision and $20(30 \%)$ received supervision in small groups but the intervention delivery (one-on-one or small group supervision) was unclear in the remaining 29 $(43 \%)$ intervention groups. Participants in most intervention groups $(71 ; 79 \%)$ were required to attend a facility for all or the majority of the intervention sessions.

3.3. Retention, Adherence, and Adverse Events. Retention was generally well reported and was high, with $62(69 \%)$ of the 90 intervention groups retaining at least $85 \%$ of participants (Table 1). Seventeen (32\%) of the 53 included trials reported that at least one participant dropped out for a reason related to the intervention (Table 2 ). Difficulties with transport and disinterest/poor adherence were the most common intervention-related reasons for dropouts.

Overall, adherence and adverse events were infrequently reported in the included trials (Table 1 ). Adherence was reported in some form in $26(49 \%)$ of the included trials. However, 11 (42\%) of these trials only reported adherence for those participants who completed the intervention. Most trials $(38 ; 72 \%)$ did not report monitoring for adverse events. Across the remaining 15 trials, 11 adverse events occurred (Table 1). Four participants from two separate trials [41, 80] experienced cardiac problems. Two of these participants, one from each group in a trial comparing physical therapy with and without mental practice [80], withdrew from the study. The two participants from the other trial [41] were able to continue safely with treadmill training. Other adverse events reported included a fall [81] and muscle cramps and tiredness [43] in trials involving cued overground walking, knee pain during a dancing program [52], muscle soreness 
Parkinson's Disease

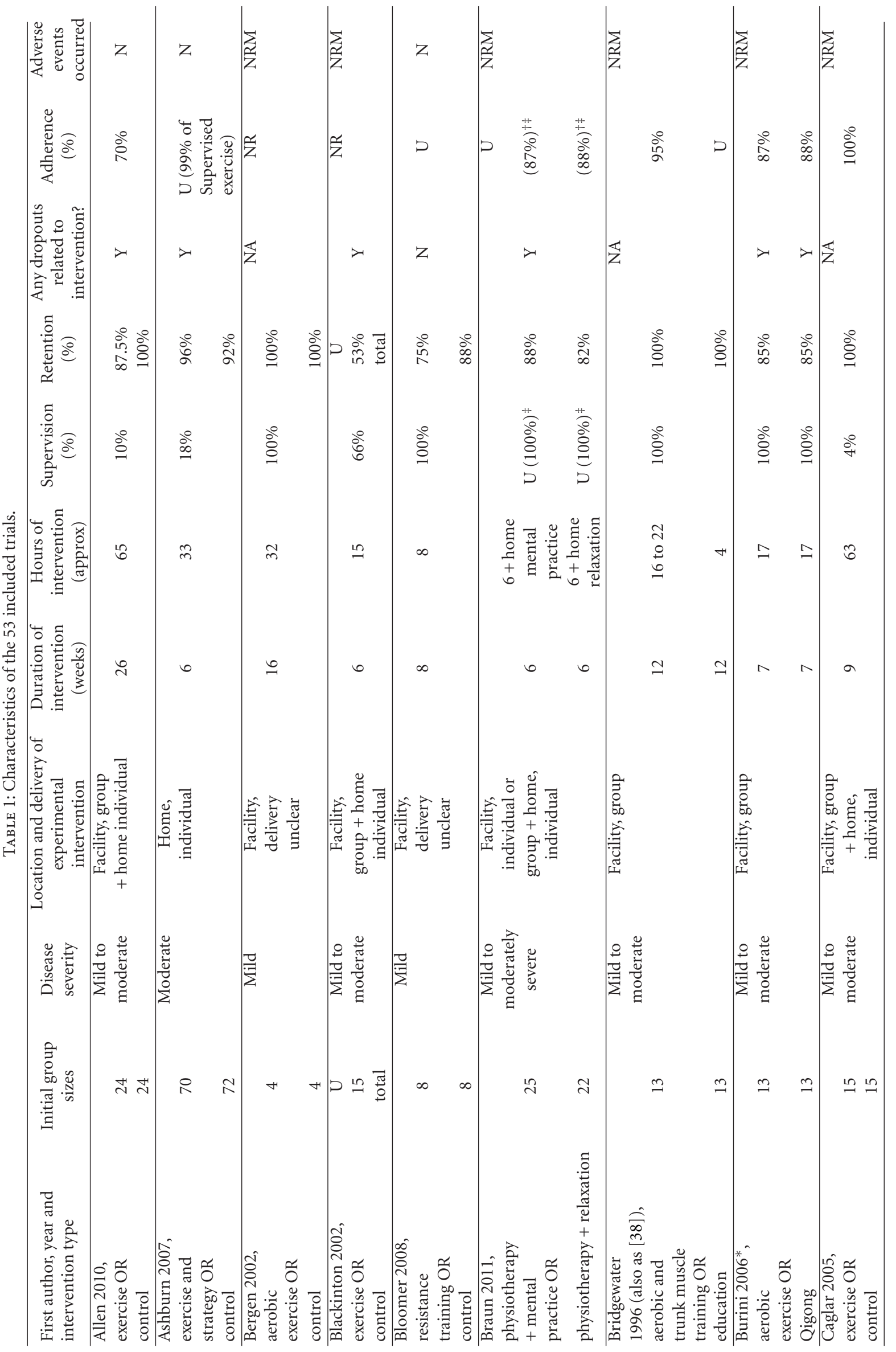




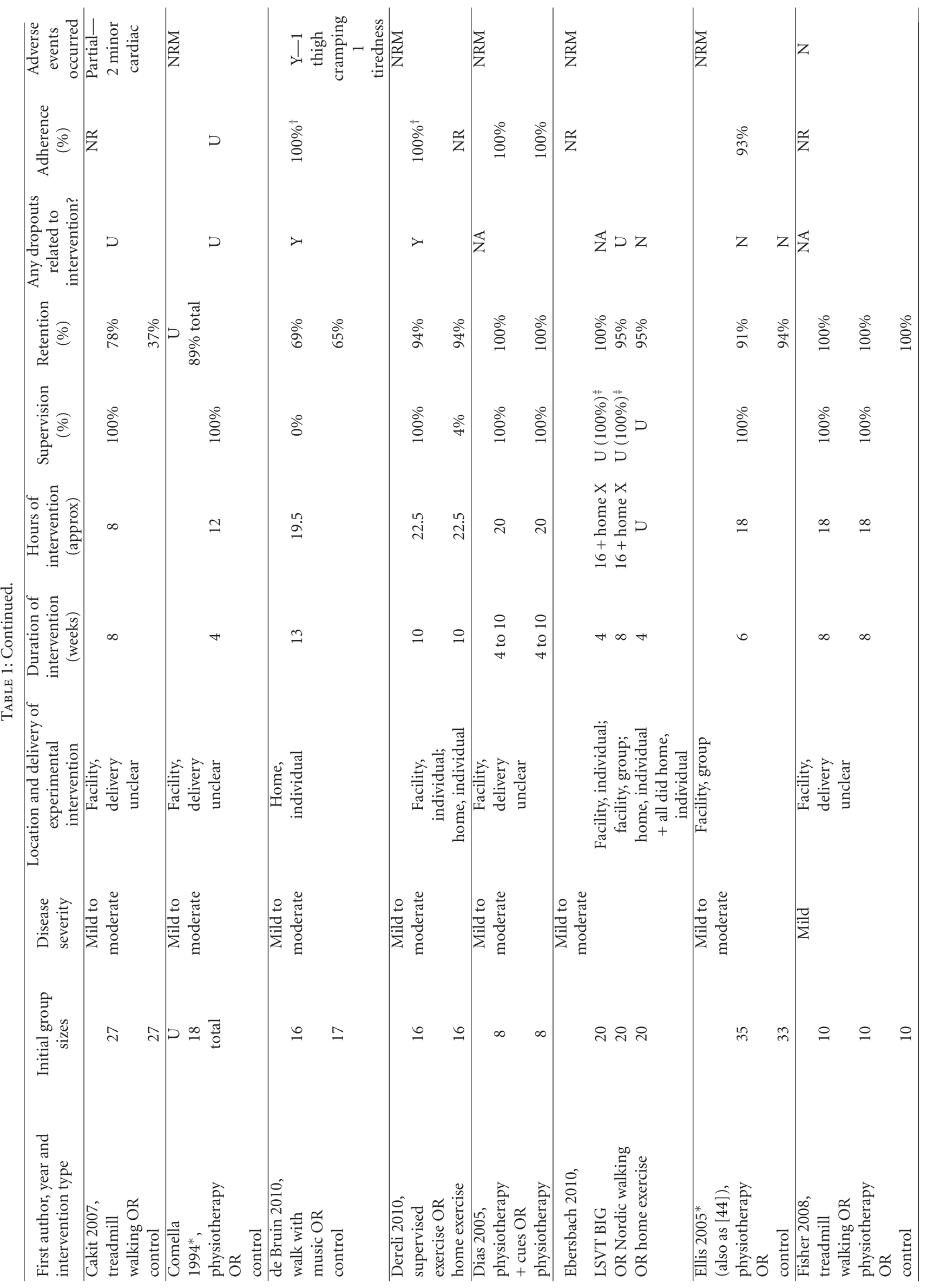




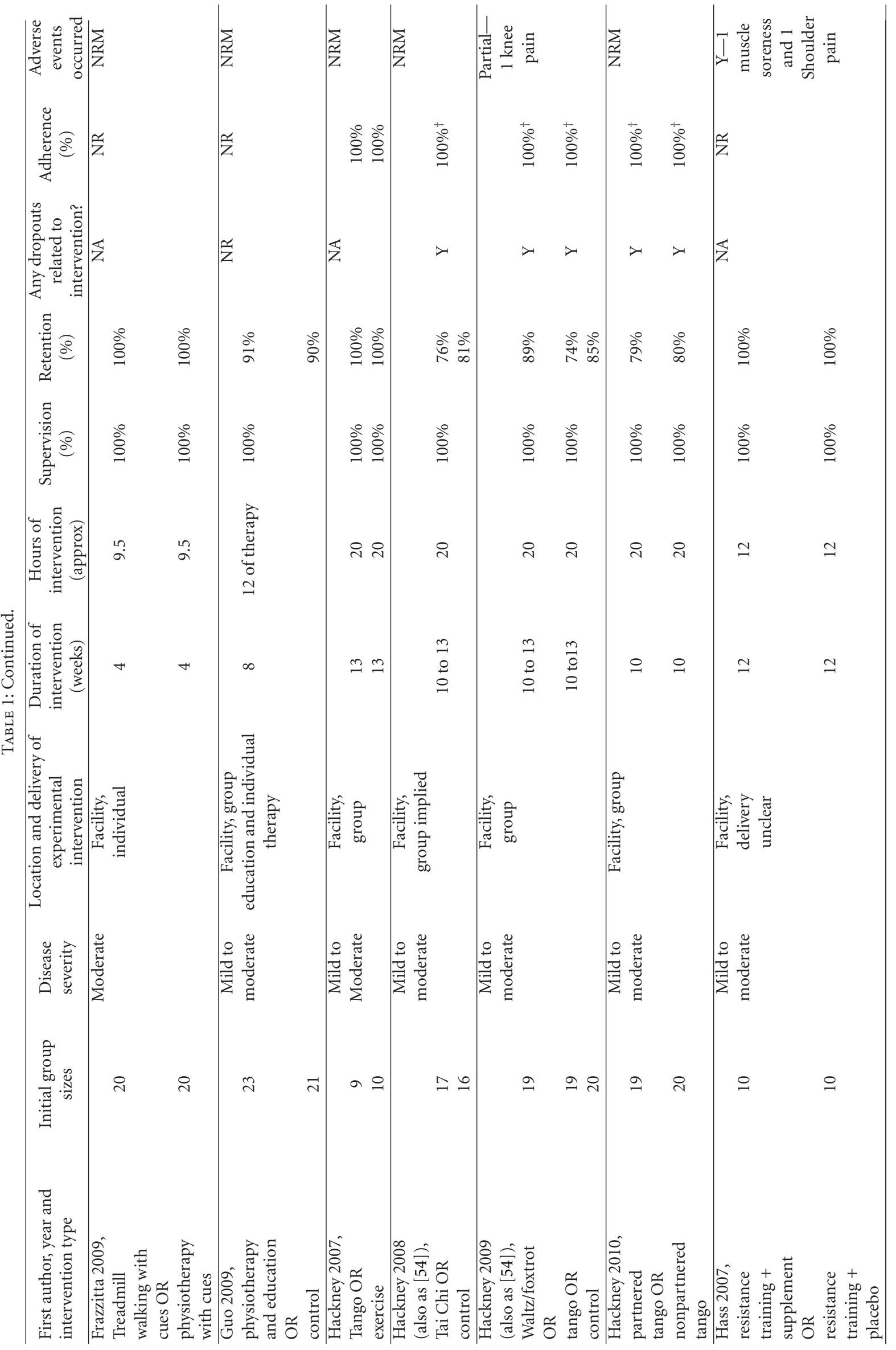




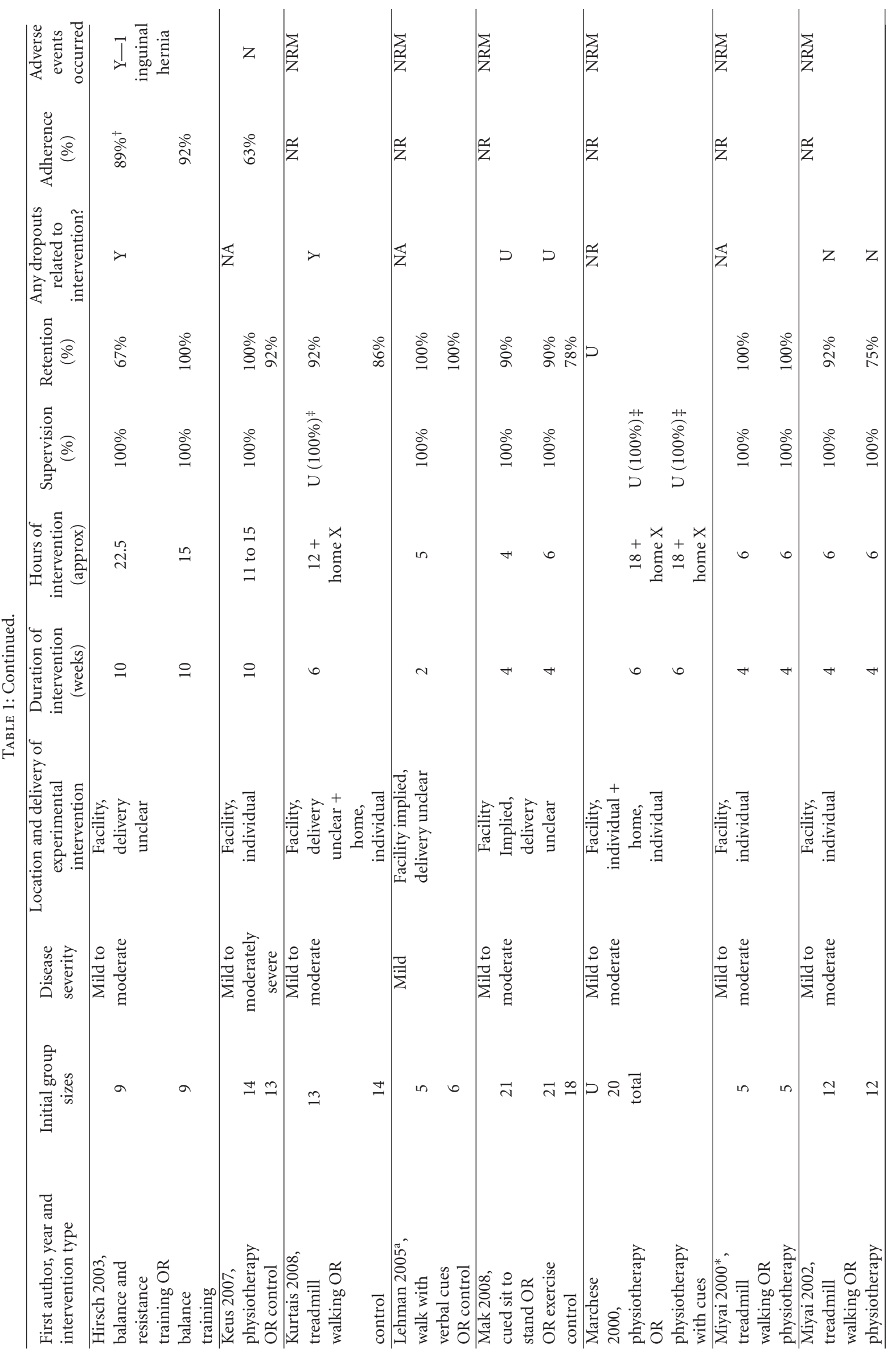




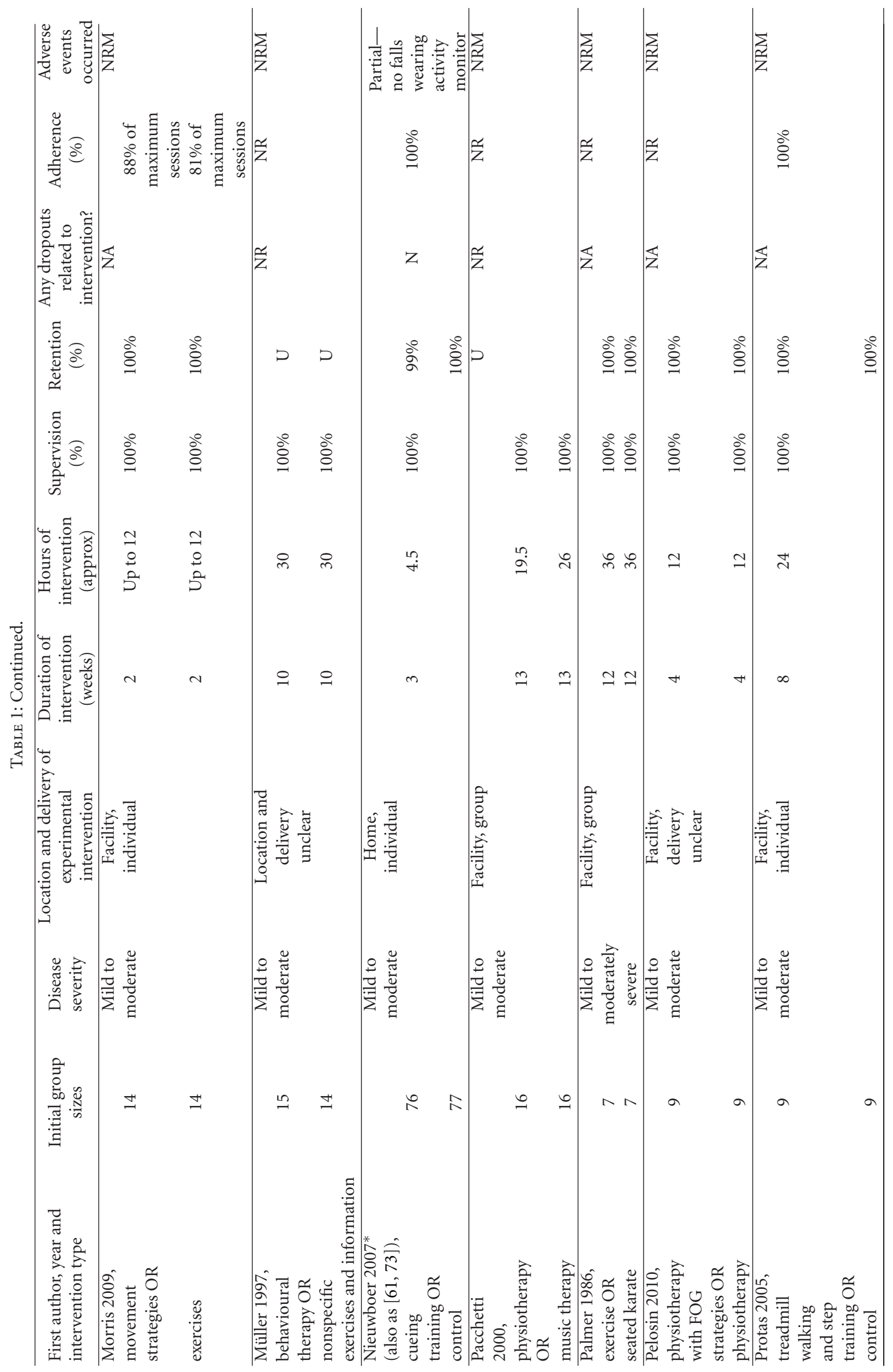




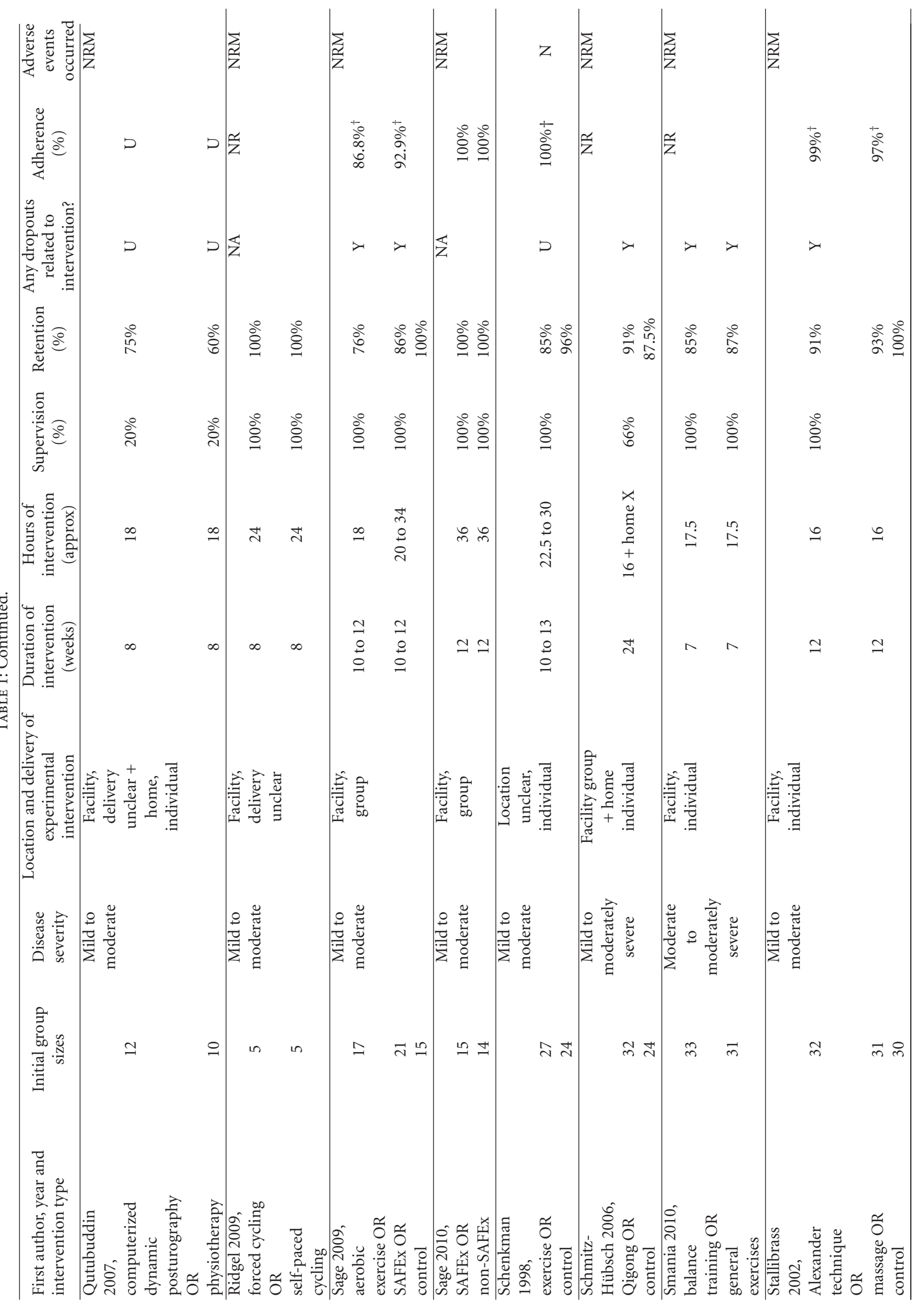




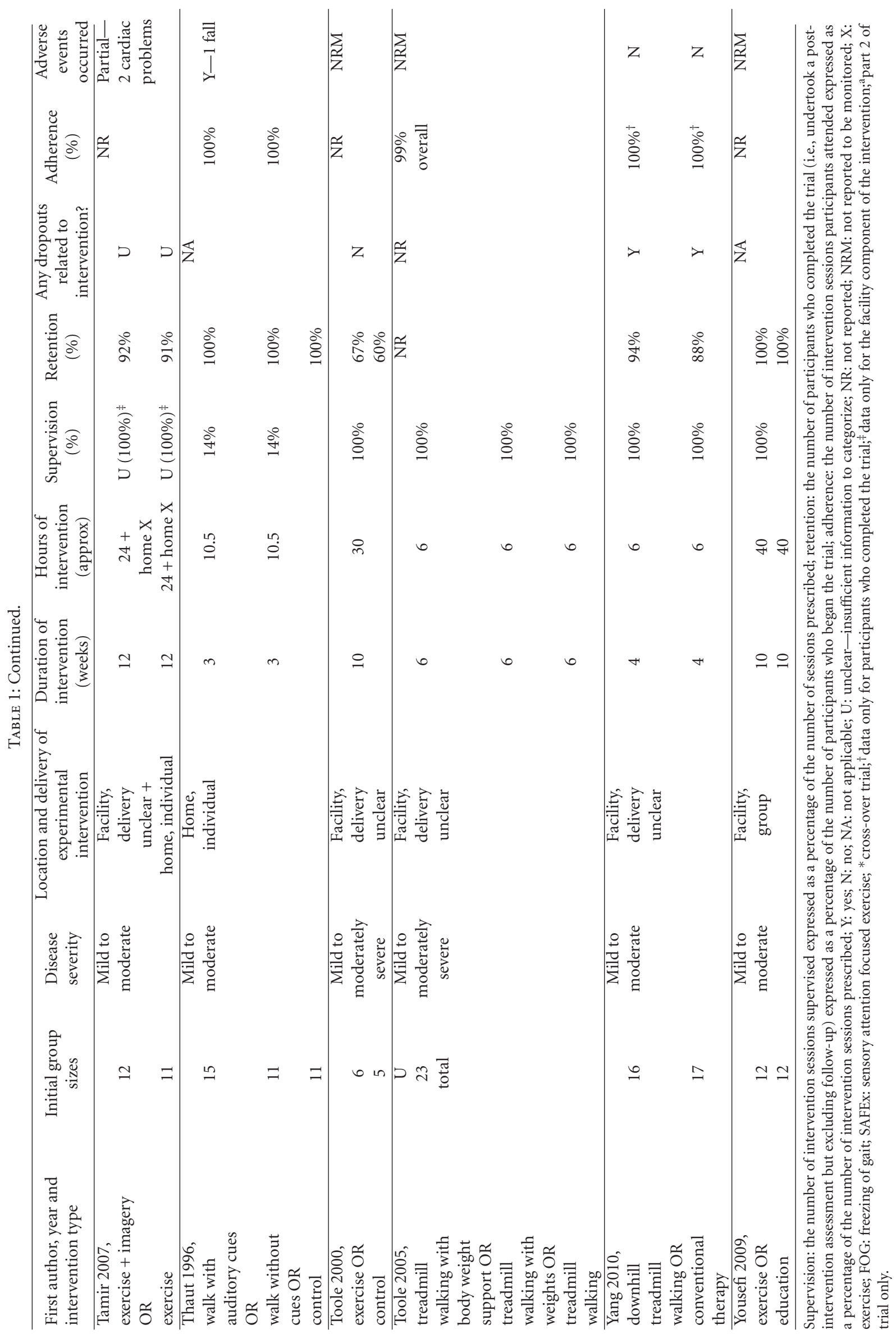


TABLE 2: Dropout reasons when related to the intervention.

\begin{tabular}{|c|c|c|}
\hline First author and year & Dropout reason & $\begin{array}{l}\text { Number of } \\
\text { participants }\end{array}$ \\
\hline Allen 2010 & Did not want to do the intervention & 1 \\
\hline Ashburn 2007 & Falls (but not during intervention) & 1 \\
\hline Blackinton 2002 & Safety concerns & 1 \\
\hline Braun 2011 & Imagery too confronting & 1 \\
\hline \multirow{2}{*}{ Burini 2006} & Poor adherence to exercise; & 2 \\
\hline & back pain & 1 \\
\hline de Bruin 2010 & No access to necessary equipment & 1 \\
\hline Dereli 2010 & Did not want to do the intervention & 1 \\
\hline \multirow{2}{*}{ Hackney 2008 (also as [54]) } & Exercise not intense enough; & 1 \\
\hline & transport problems & 2 \\
\hline \multirow{2}{*}{ Hackney 2009 (also as [54]) } & Knee pain; & 1 \\
\hline & transport problems & 2 \\
\hline \multirow{3}{*}{ Hackney 2010} & Travel distance; & 2 \\
\hline & classes too fatiguing; & 1 \\
\hline & lack of interest & 1 \\
\hline Hirsch 2003 & Inguinal hernia & 1 \\
\hline Kurtais 2008 & Poor adherence to exercise & 1 \\
\hline Sage 2009 & Time commitment & 4 \\
\hline \multirow{2}{*}{ Schmitz-Hubsch 2006} & Uncomfortable in the group; & 1 \\
\hline & uncomfortable with Qigong & 1 \\
\hline Smania 2010 & Uncooperative & 4 \\
\hline Stallibrass 2002 & Could not travel & 1 \\
\hline \multirow{2}{*}{ Yang 2010} & Low motivation; & 1 \\
\hline & transport problems & 1 \\
\hline
\end{tabular}

and shoulder pain [56] following resistance training, and a hernia [57] subsequent to muscle strength assessment.

\section{Discussion}

A substantial number of randomized controlled trials of exercise and/or motor training for people with PD have been published. However, the nature and reporting of these trials are likely to provide challenges for therapists aiming to implement the interventions into clinical practice [17]. Most trials involved only cognitively intact participants with mildto-moderate PD. Trials tended to be of short duration, highly supervised, and conducted at a facility. Furthermore, the reports for many trials were lacking important details, with adherence and adverse events particularly being inadequately reported.

On the whole, trials included in this paper included only participants with mild-to-moderate PD who were without significant cognitive impairment. Including only these types of participants not only makes it easier to conduct trials of exercise and motor training interventions but also aids interpretation of the results. However, cognitive impairment is now recognised as a common problem in $\mathrm{PD}$, with over $80 \%$ of people with PD ultimately developing dementia [89]. Further work is needed to determine the effectiveness of exercise and motor training in people with more severe cognitive impairment and/or more advanced disease.

Most of the reviewed trials were of short duration, highly supervised, and facility based (Table 1). Interventions lasted an average of around two months. Seventy-four percent of the intervention groups were fully supervised, with no reported expectation for participants to undertake unsupervised exercise. Furthermore, 79\% of intervention groups were mainly conducted at a facility such as a hospital or university. Such brief, highly supervised interventions conducted in controlled environments are likely to improve the adherence of participants to exercise and motor training programs and to ensure that interventions are being performed optimally. In this regard, these trials are useful and important for determining the short-term efficacy of an intervention. However, given that PD is a long-term, neurodegenerative condition, the capacity of therapists and patients to sustain the intervention over the long term needs to be considered. Furthermore, such brief and highly supervised interventions are costly and less likely to give 
information about the effectiveness of the intervention when implemented into usual practice $[13,17]$. For example, the requirement for participants to travel to a facility was a common reason for withdrawal from the included trials (Table 2). Moreover, the neurodegenerative nature of PD and the limited resources available to healthcare systems mean that such labor-intensive programs are unlikely to be sustained or afforded by most health-care providers. Additionally, as PD is a progressive disease it is important that people with PD are empowered to self-manage their disease to some extent $[90,91]$. To this end, trials of more pragmatic and sustainable exercise and motor training interventions, with the potential for direct translation into clinical practice and including cost-effectiveness analysis, are needed.

The likely adherence to an exercise and motor training program is an important factor to consider when prescribing such a program for an individual with PD. Adherence to the intervention was reported in less than half of the included trials, and some reports of adherence are artificially elevated by including only those participants who completed the trial (Table 1). Some trials were able to effectively maximise adherence by providing a flexible timeframe for participants to complete the intervention $[46,51,52,74,76]$ and so allow participants more options in fitting their exercise and/or motor training program around their daily lives. This pragmatic approach is likely to more closely reflect therapy attendance patterns and is therefore likely to be helpful for therapists considering translating the research into their clinical practice.

Given the importance of adherence to exercise and motor training programs, strategies to promote adherence in people with PD need to be considered. Providing a high level of supervision seems likely to promote adherence in the short term, as it may enhance participants' commitment to the program. However, a Cochrane review comparing home and centre-based exercise programs for older adults found that, in the long term, participants were more likely to adhere to home-based programs [92]. Furthermore, the reviewers noted a trend toward more sustained improvements in the home-based than in the centre-based programs and suggested that this was attributable to the higher adherence in home-based programs. In the present paper, three of the included trials report high levels of adherence with minimally supervised home-based programs [40, 43, 81]. Common to all three of these trials was a requirement for participants to keep a daily record of what exercise/motor training they had performed. It seems likely that this simple strategy assisted in promoting adherence in these trials. Other strategies with the potential to improve adherence in sustainable, minimally supervised trials, such as participant involvement in goal setting $[93,94]$, flexibility to allow programs to be modified for individuals [1, 91, 93, 94], and intermittent followup [91, 94], warrant exploration.

The issue of adverse events was inadequately addressed in the trials included in this paper, with only 15 trials reporting monitoring for adverse events. In these 15 trials, 11 adverse events were reported, most of which were minor in nature (Table 1). However, when discussing and planning exercise and motor training options with people with PD, therapists need to be informed not only about the effectiveness of a given intervention but also about the nature and likelihood of any potential adverse events [95]. Similarly poor reporting of adverse events was found in a recent Cochrane review of progressive resistance training for older adults [95]. Notably, the Cochrane review found that adverse events were more likely to be detected in trials that used a clear definition of adverse events than in trials which did not use a definition. In the same way, the use of a definition for adverse events is likely to improve the assessment and reporting of adverse events in trials of exercise and motor training for people with PD.

This paper has examined several factors in the nature and reporting of trials of exercise and/or motor training which are likely to influence the way research is applied by therapists in clinical practice. However, this paper did not address whether or not trial protocols were reported in sufficient detail to allow therapists to emulate the research intervention in the clinic. This detailed reporting of trial interventions is critical in enabling research to be clinically applied [96]. The ability of many journals to provide online material which supplements the published article will aid the provision of such details despite the necessary word count limitations placed on authors.

\section{Conclusions}

Clinicians seeking to use research to inform their clinical practice rely heavily on the design and reporting of randomized controlled trials to reach their decisions. However, the nature and reporting of trials of exercise and/or motor training for people with PD are likely to provide challenges for therapists aiming to implement the interventions into clinical practice. The short duration, highly supervised and facility-based nature of many of the interventions, coupled with the tendency to include only cognitively-intact participants with mild-to-moderate disease, mean that findings may not generalise when therapists set out to apply them in the long-term management of people with $\mathrm{PD}$. Infrequent reporting of adherence and adverse events compounds this problem and makes cost-benefit balancing more difficult. It is recommended that these issues be taken into account in the design and reporting of future trials.

\section{References}

[1] N. E. Allen, C. G. Canning, C. Sherrington et al., "The effects of an exercise program on fall risk factors in people with Parkinson's disease: a randomized controlled trial," Movement Disorders, vol. 25, no. 9, pp. 1217-1225, 2010.

[2] N. E. Allen, C. Sherrington, S. S. Paul, and C. G. Canning, "Balance and falls in Parkinson's disease: a meta-analysis of the effect of exercise and motor training," Movement Disorders, vol. 26, no. 9, pp. 1605-1615, 2001.

[3] A. Ashburn, L. Fazakarley, C. Ballinger, R. Pickering, L. D. McLellan, and C. Fitton, "A randomised controlled trial of a home based exercise programme to reduce the risk of falling among people with Parkinson's disease," Journal of Neurology, Neurosurgery and Psychiatry, vol. 78, no. 7, pp. 678-684, 2007. 
[4] C. J. de Goede, S. H. J. Keus, G. Kwakkel, and R. C. Wagenaar, "The effects of physical therapy in Parkinson's disease: a research synthesis," Archives of Physical Medicine and Rehabilitation, vol. 82, no. 4, pp. 509-515, 2001.

[5] L. E. Dibble, O. Addison, and E. Papa, "The effects of exercise on balance in persons with parkinson's disease: a systematic review across the disability spectrum," Journal of Neurologic Physical Therapy, vol. 33, no. 1, pp. 14-26, 2009.

[6] G. Ebersbach, A. Ebersbach, D. Edler et al., "Comparing exercise in Parkinson's disease-the Berlin BIG study," Movement Disorders, vol. 25, no. 12, pp. 1902-1908, 2010.

[7] G. Ebersbach, A. Ebersbach, D. Edler et al., "Comparing exercie in Parkinson's disease-the Berlin LSVT®BIG study," Movement Disorders, vol. 25, no. 14, p. 2478, 2010.

[8] V. A. Goodwin, S. H. Richards, R. S. Taylor, A. H. Taylor, and J. L. Campbell, "The effectiveness of exercise interventions for people with Parkinson's disease: a systematic review and meta-analysis," Movement Disorders, vol. 23, no. 5, pp. 631-640, 2008.

[9] J. Mehrholz, R. Friis, J. Kugler, S. Twork, A. Storch, and M. Pohl, "Treadmill training for patients with Parkinson's disease," Cochrane Database of Systematic Reviews, no. 1, Article ID CD007830, 2010.

[10] M. E. Morris, R. Iansek, and B. Kirkwood, "A randomized controlled trial of movement strategies compared with exercise for people with Parkinson's disease," Movement Disorders, vol. 24, no. 1, pp. 64-71, 2009.

[11] A. Nieuwboer, G. Kwakkel, L. Rochester et al., "Cueing training in the home improves gait-related mobility in Parkinson's disease: The RESCUE trial," Journal of Neurology, Neurosurgery and Psychiatry, vol. 78, no. 2, pp. 134-140, 2007.

[12] R. C. Brownson and E. Jones, "Bridging the gap: translating research into policy and practice," Preventive Medicine, vol. 49, no. 4, pp. 313-315, 2009.

[13] B. Haynes and A. Haines, "Getting research findings into practice. Barriers and bridges to evidence based clinical practice," British Medical Journal, vol. 317, no. 7153, pp. 273-276, 1998.

[14] R. Herbert, G. Jamtvedt, J. Mead, and K. Birger Hagen, Practical Evidence-Based Physiotherapy, Elsevier, Edinburgh, UK, 2005.

[15] E. V. Cyarto, W. J. Brown, and A. L. Marshall, "Retention, adherence and compliance: important considerations for home- and group-based resistance training programs for older adults," Journal of Science and Medicine in Sport, vol. 9, no. 5, pp. 402-412, 2006.

[16] L. Quinn, M. Busse, H. Khalil, S. Richardson, A. Rosser, and H. Morris, "Client and therapist views on exercise programmes for early-mid stage Parkinson's disease and Huntington's disease," Disability and Rehabilitation, vol. 32, no. 11, pp. 917-928, 2010.

[17] T. A. Sheldon, G. H. Guyatt, and A. Haines, "Getting research findings into practice. When to act on the evidence," British Medical Journal, vol. 317, no. 7151, pp. 139-142, 1998.

[18] A. M. Crizzle and I. J. Newhouse, "Is physical exercise beneficial for persons with Parkinson's disease?" Clinical Journal of Sport Medicine, vol. 16, no. 5, pp. 422-425, 2006.

[19] K. H. Deane, D. Jones, C. Ellis-Hill, C. E. Clarke, E. D. Playford, and Y. Ben-Shlomo, "A comparison of physiotherapy techniques for patients with Parkinson's disease," Cochrane Database of Systematic Reviews, no. 1, Article ID CD002815, 2001.

[20] K. Deane, D. Jones, E. Playford, Y. Ben-Shlomo, and C. Clarke, "Physiotherapy versus placebo or no intervention in
Parkinson's disease," Cochrane Database of Systematic Reviews, no. 3, Article ID CD002817, 2001.

[21] K. H. O. Deane, C. Ellis-Hill, D. Jones et al., "Systematic review of paramedical therapies for Parkinson's disease," Movement Disorders, vol. 17, no. 5, pp. 984-991, 2002.

[22] H. Gage and L. Storey, "Rehabilitation for Parkinson's disease: a systematic review of available evidence," Clinical Rehabilitation, vol. 18, no. 5, pp. 463-482, 2004.

[23] M. Jöbges, H. Spittler-Schneiders, C. I. E. Renner, and H. Hummelsheim, "Clinical relevance of rehabilitation programs for Parkinson's disease. I: non-symptom-specific therapeutic approaches," Parkinsonism and Related Disorders, vol. 13, no. 4, pp. 195-202, 2007.

[24] E. M. Jöbges, H. Spittler-Schneiders, C. I. E. Renner, and H. Hummelsheim, "Clinical relevance of rehabilitation programs for patients with idiopathic Parkinson syndrome. II: symptom-specific therapeutic approaches," Parkinsonism and Related Disorders, vol. 13, no. 4, pp. 203-213, 2007.

[25] S. H. J. Keus, B. R. Bloem, E. J. M. Hendriks, A. B. BrederoCohen, and M. Munneke, "Evidence-based analysis of physical therapy in Parkinson's disease with recommendations for practice and research," Movement Disorders, vol. 22, no. 4, pp. 451-460, 2007.

[26] G. Kwakkel, C. J. T. de Goede, and E. E. H. van Wegen, "Impact of physical therapy for Parkinson's disease: a critical review of the literature," Parkinsonism and Related Disorders, vol. 13, supplement 3, pp. S478-S487, 2007.

[27] I. Lim, E. van Wegen, C. de Goede et al., "Effects of external rhythmical cueing on gait in patients with Parkinson's disease: a systematic review," Clinical Rehabilitation, vol. 19, no. 7, pp. 695-713, 2005.

[28] A. Nieuwboer, "Cueing for freezing of gait in patients with Parkinson's disease: a rehabilitation perspective," Movement Disorders, vol. 23, supplement 2, pp. S475-S481, 2008.

[29] T. C. Rubinstein, N. Giladi, and J. M. Hausdorff, "The power of cueing to circumvent dopamine deficits: a review of physical therapy treatment of gait disturbances in Parkinson's disease," Movement Disorders, vol. 17, no. 6, pp. 1148-1160, 2002.

[30] O. Suchowersky, G. Gronseth, J. Perlmutter, S. Reich, T. Zesiewicz, and W. J. Weiner, "Practice parameter: Neuroprotective strategies and alternative therapies for Parkinson disease (an evidence-based review)," Neurology, vol. 66, no. 7, pp. 976-982, 2006.

[31] J. P. T. Higgins and S. Green, Cochrane Handbook for Systematic Reviews of Interventions Version 5.1.0, The Cochrane Collaboration, 2009.

[32] A. Liberati, D. G. Altman, J. Tetzlaff et al., "The PRISMA statement for reporting systematic reviews and meta-analyses of studies that evaluate health care interventions: explanation and elaboration," Journal of Clinical Epidemiology, vol. 62, no. 10, pp. e1-e34, 2009.

[33] J. L. Bergen, T. Toole, R. G. Elliott Jr., B. Wallace, K. Robinson, and C. G. Maitland, "Aerobic exercise intervention improves aerobic capacity and movement initiation in Parkinson's disease patients," NeuroRehabilitation, vol. 17, no. 2, pp. 161-168, 2002.

[34] M. Blackinton, L. Summerall, and K. Waguespack, "Tertiary prevention in Parkinson disease: results from a preliminary study," Neurology Report, vol. 26, no. 3, pp. 160-165, 2002.

[35] R. J. Bloomer, B. K. Schilling, R. E. Karlage, M. S. Ledoux, R. F. Pfeiffer, and J. Callegari, "Effect of resistance training on blood oxidative stress in Parkinson disease," Medicine and Science in Sports and Exercise, vol. 40, no. 8, pp. 1385-1389, 2008. 
[36] S. Braun, A. Beurskens, M. Kleynen, J. Schols, and D. Wade, "Rehabilitation with mental practice has similar effects on mobility as rehabilitation with relaxation in people with Parkinson's disease: a multicentre randomised trial," Journal of Physiotherapy, vol. 57, no. 1, pp. 27-34, 2011.

[37] K. J. Bridgewater and M. H. Sharpe, "Aerobic exercise and early parkinson's disease," Neurorehabilitation and Neural Repair, vol. 10, no. 4, pp. 233-241, 1996.

[38] K. J. Bridgewater and M. H. Sharpe, "Trunk muscle training and early Parkinson's disease," Physiotherapy Theory and Practice, vol. 13, no. 2, pp. 139-153, 1997.

[39] D. Burini, B. Farabollini, S. Iacucci et al., "A randomised controlled cross-over trial of aerobic training versus Qigong in advanced Parkinson's disease," Europa Medicophysica, vol. 42, no. 3, pp. 231-238, 2006.

[40] A. T. Caglar, H. N. Gurses, F. K. Mutluay, and G. Kiziltan, "Effects of home exercises on motor performance in patients with Parkinson's disease," Clinical Rehabilitation, vol. 19, no. 8, pp. 870-877, 2005.

[41] B. D. Cakit, M. Saracoglu, H. Genc, H. R. Erdem, and L. Inan, "The effects of incremental speed-dependent treadmill training on postural instability and fear of falling in Parkinson's disease," Clinical Rehabilitation, vol. 21, no. 8, pp. 698-705, 2007.

[42] C. L. Comella, G. T. Stebbins, N. Brown-Toms, and C. G. Goetz, "Physical therapy and Parkinson's disease: a controlled clinical trial," Neurology, vol. 44, no. 3, part 1, pp. 376-378, 1994.

[43] N. de Bruin, J. B. Doan, G. Turnbull et al., "Walking with music is a safe and viable tool for gait training in parkinson's disease: the effect of a 13-week feasibility study on single and dual task walking," Parkinson's Disease, vol. 2010, Article ID 483530, 9 pages, 2010.

[44] C. J. T. de Goede, T. Ellis, and R. C. Wagenaar, "Effecten van een fysiotherapie-groepsbehandeling Parkinson-patienten: een cross-over trial," Nederlands Tijdschrift voor Fysiotherapie, vol. 114, no. 3, pp. 78-82, 2004.

[45] E. E. Dereli and A. Yaliman, "Comparison of the effects of a physiotherapist-supervised exercise programme and a self-supervised exercise programme on quality of life in patients with Parkinson's disease," Clinical Rehabilitation, vol. 24, no. 4, pp. 352-362, 2010.

[46] N. Dias, D. Fraga, E. Cacho, and T. Oberg, "Training of gait with visual cues in the patient with Parkinson's disease," Fisioterapia em Movimento, vol. 18, no. 4, pp. 43-51, 2005.

[47] T. Ellis, C. J. de Goede, R. G. Feldman, E. C. Wolters, G. Kwakkel, and R. C. Wagenaar, "Efficacy of a physical therapy program in patients with Parkinson's disease: a randomized controlled trial," Archives of Physical Medicine and Rehabilitation, vol. 86, no. 4, pp. 626-632, 2005.

[48] B. E. Fisher, A. D. Wu, G. J. Salem et al., "The effect of exercise training in improving motor performance and corticomotor excitability in people with early Parkinson's disease," Archives of Physical Medicine and Rehabilitation, vol. 89, no. 7, pp. 1221-1229, 2008.

[49] G. Frazzitta, R. Maestri, D. Uccellini, G. Bertotti, and P. Abelli, "Rehabilitation treatment of gait in patients with Parkinson's disease with freezing: a comparison between two physical therapy protocols using visual and auditory cues with or without treadmill training," Movement Disorders, vol. 24, no. 8, pp. 1139-1143, 2009.

[50] L. Guo, Y. Jiang, H. Yatsuya, Y. Yoshida, and J. Sakamoto, "Group education with personal rehabilitation for idiopathic Parkinson's disease," Canadian Journal of Neurological Sciences, vol. 36, no. 1, pp. 51-59, 2009.
[51] M. E. Hackney and G. M. Earhart, "Tai Chi improves balance and mobility in people with Parkinson disease," Gait and Posture, vol. 28, no. 3, pp. 456-460, 2008.

[52] M. E. Hackney and G. M. Earhart, "Effects of dance on movement control in Parkinson's disease: a comparison of Argentine tango and American ballroom," Journal of Rehabilitation Medicine, vol. 41, no. 6, pp. 475-481, 2009.

[53] M. E. Hackney and G. M. Earhart, "Effects of dance on gait and balance in Parkinsons disease: a comparison of partnered and nonpartnered dance movement," Neurorehabilitation and Neural Repair, vol. 24, no. 4, pp. 384-392, 2010.

[54] M. E. Hackney and G. M. Earhart, "Health-related quality of life and alternative forms of exercise in Parkinson disease," Parkinsonism and Related Disorders, vol. 15, no. 9, pp. 644-648, 2009.

[55] M. E. Hackney, S. Kantorovich, R. Levin, and G. M. Earhart, "Effects of tango on functional mobility in Parkinson's disease: a preliminary study," Journal of Neurologic Physical Therapy, vol. 31, no. 4, pp. 173-179, 2007.

[56] C. J. Hass, M. A. Collins, and J. L. Juncos, "Resistance training with creatine monohydrate improves upper-body strength in patients with Parkinson disease: a randomized trial," Neurorehabilitation and Neural Repair, vol. 21, no. 2, pp. 107-115, 2007.

[57] M. A. Hirsch, T. Toole, C. G. Maitland, and R. A. Rider, "The effects of balance training and high-intensity resistance training on persons with idiopathic Parkinson's disease," Archives of Physical Medicine and Rehabilitation, vol. 84, no. 8, pp. 1109-1117, 2003.

[58] S. H. J. Keus, B. R. Bloem, J. J. van Hilten, A. Ashburn, and M. Munneke, "Effectiveness of physiotherapy in Parkinson's disease: the feasibility of a randomised controlled trial," Parkinsonism and Related Disorders, vol. 13, no. 2, pp. 115-121, 2007.

[59] Y. Kurtais, S. Kutlay, B. S. Tur, H. Gok, and C. Akbostanci, "Does treadmill training improve lower-extremity tasks in Parkinson disease? A randomized controlled trial," Clinical Journal of Sport Medicine, vol. 18, no. 3, pp. 289-291, 2008.

[60] D. A. Lehman, T. Toole, D. Lofald, and M. A. Hirsch, "Training with verbal instructional cues results in near-term improvement of gait in people with Parkinson disease," Journal of Neurologic Physical Therapy, vol. 29, no. 1, pp. 2-8, 2005.

[61] I. Lim, E. van Wegen, D. Jones et al., "Does cueing training improve physical activity in patients with parkinson's disease?" Neurorehabilitation and Neural Repair, vol. 24, no. 5, pp. 469-477, 2010.

[62] M. K. Y. Mak and C. W. Y. Hui-Chan, "Cued task-specific training is better than exercise in improving sit-to-stand in patients with Parkinson's disease: a randomized controlled trial," Movement Disorders, vol. 23, no. 4, pp. 501-509, 2008.

[63] R. Marchese, M. Diverio, F. Zucchi, C. Lentino, and G. Abbruzzese, "The role of sensory cues in the rehabilitation of Parkinsonian patients: a comparison of two physical therapy protocols," Movement Disorders, vol. 15, no. 5, pp. 879-883, 2000.

[64] I. Miyai, Y. Fujimoto, Y. Ueda et al., "Treadmill training with body weight support: its effect on Parkinson's disease," Archives of Physical Medicine and Rehabilitation, vol. 81, no. 7, pp. 849-852, 2000.

[65] I. Miyai, Y. Fujimoto, H. Yamamoto et al., "Long-term effect of body weight-supported treadmill training in Parkinson's disease: a randomized controlled trial," Archives of Physical Medicine and Rehabilitation, vol. 83, no. 10, pp. 1370-1373, 2002. 
[66] V. Müller, B. Mohr, R. Rosin, F. Pulvermüller, F. Müller, and N. Birbaumer, "Short-term effects of behavioral treatment on movement initiation and postural control in Parkinson's disease: a controlled clinical study," Movement Disorders, vol. 12, no. 3, pp. 306-314, 1997.

[67] C. Pacchetti, F. Mancini, R. Aglieri, C. Fundaró, E. Martignoni, and G. Nappi, "Active music therapy in Parkinson's disease: an integrative method for motor and emotional rehabilitation," Psychosomatic Medicine, vol. 62, no. 3, pp. 386-393, 2000.

[68] S. S. Palmer, J. A. Mortimer, D. D. Webster, R. Bistevins, and G. L. Dickinson, "Exercise therapy for Parkinson's disease," Archives of Physical Medicine and Rehabilitation, vol. 67, no. 10, pp. 741-745, 1986.

[69] E. Pelosin, L. Avanzino, M. Bove, P. Stramesi, A. Nieuwboer, and G. Abbruzzese, "Action observation improves freezing of gait in patients with Parkinson's disease," Neurorehabilitation and Neural Repair, vol. 24, no. 8, pp. 746-752, 2010.

[70] E. J. Protas, K. Mitchell, A. Williams, H. Qureshy, K. Caroline, and E. C. Lai, "Gait and step training to reduce falls in Parkinson's disease," NeuroRehabilitation, vol. 20, no. 3, pp. 183-190, 2005.

[71] A. A. Qutubuddin, D. X. Cifu, P. Armistead-Jehle, W. Carne, T. E. McGuirk, and M. S. Baron, "A comparison of computerized dynamic posturography therapy to standard balance physical therapy in individuals with Parkinson's disease: a pilot study," NeuroRehabilitation, vol. 22, no. 4, pp. 261-265, 2007.

[72] A. L. Ridgel, J. L. Vitek, and J. L. Alberts, "Forced, not voluntary, exercise improves motor function in Parkinson's disease patients," Neurorehabilitation and Neural Repair, vol. 23, no. 6, pp. 600-608, 2009.

[73] L. Rochester, K. Baker, V. Hetherington et al., "Evidence for motor learning in Parkinson's disease: acquisition, automaticity and retention of cued gait performance after training with external rhythmical cues," Brain Research, vol. 1319, pp. 103-111, 2010.

[74] M. D. Sage and Q. J. Almeida, "Symptom and gait changes after sensory attention focused exercise vs aerobic training in Parkinson's disease," Movement Disorders, vol. 24, no. 8, pp. 1132-1138, 2009.

[75] M. D. Sage and Q. J. Almeida, "A positive influence of vision on motor symptoms during sensory attention focused exercise for Parkinson's disease," Movement Disorders, vol. 25, no. 1, pp. 64-69, 2010.

[76] M. Schenkman, T. M. Cutson, M. Kuchibhatla et al., "Exercise to improve spinal flexibility and function for people with Parkinson's disease: a randomized, controlled trial," Journal of the American Geriatrics Society, vol. 46, no. 10, pp. 1207-1216, 1998.

[77] T. Schmitz-Hübsch, D. Pyfer, K. Kielwein, R. Fimmers, T. Klockgether, and U. Wüllner, "Qigong exercise for the symptoms of Parkinson's disease: a randomized, controlled pilot study," Movement Disorders, vol. 21, no. 4, pp. 543-548, 2006.

[78] N. Smania, E. Corato, M. Tinazzi et al., "Effect of balance training on postural instability in patients with idiopathic parkinsong's disease," Neurorehabilitation and Neural Repair, vol. 24, no. 9, pp. 826-834, 2010.

[79] C. Stallibrass, P. Sissons, and C. Chalmers, "Randomized controlled trial of the Alexander Technique for idiopathic Parkinson's disease," Clinical Rehabilitation, vol. 16, no. 7, pp. 695-708, 2002.

[80] R. Tamir, R. Dickstein, and M. Huberman, "Integration of motor imagery and physical practice in group treatment applied to subjects with Parkinson's disease," Neurorehabilitation and Neural Repair, vol. 21, no. 1, pp. 68-75, 2007.
[81] M. H. Thaut, G. C. McIntosh, R. R. Rice, R. A. Miller, J. Rathbun, and J. M. Brault, "Rhythmic auditory stimulation in gait training for Parkinson's disease patients," Movement Disorders, vol. 11, no. 2, pp. 193-200, 1996.

[82] T. Toole, M. A. Hirsch, A. Forkink, D. A. Lehman, and C. G. Maitland, "The effects of a balance and strength training program on equilibrium in Parkinsonism: a preliminary study," NeuroRehabilitation, vol. 14, no. 3, pp. 165-174, 2000.

[83] T. Toole, C. G. Maitland, E. Warren, M. F. Hubmann, and L. Panton, "The effects of loading and unloading treadmill walking on balance, gait, fall risk, and daily function in Parkinsonism," NeuroRehabilitation, vol. 20, no. 4, pp. 307-322, 2005.

[84] Y. R. Yang, Y. Y. Lee, S. J. Cheng, and R. Y. Wang, "Downhill walking training in individuals with parkinson's disease: a randomized controlled trial," American Journal of Physical Medicine and Rehabilitation, vol. 89, no. 9, pp. 706-714, 2010.

[85] B. Yousefi, V. Tadibi, A. F. Khoei, and A. Montazeri, "Exercise therapy, quality of life, and activities of daily living in patients with Parkinson disease: a small scale quasi-randomised trial," Trials, vol. 10, article 67, 2009.

[86] M. M. Hoehn and M. D. Yahr, "Parkinsonism: onset, progression and mortality," Neurology, vol. 17, no. 5, pp. 427-442, 1967.

[87] M. F. Folstein, S. E. Folstein, and P. R. McHugh, “'Mini mental state'. A practical method for grading the cognitive state of patients for the clinician," Journal of Psychiatric Research, vol. 12, no. 3, pp. 189-198, 1975.

[88] M. E. Oehlert, S. D. Hass, M. R. Freeman, M. D. Williams, J. J. Ryan, and S. W. Sumerall, "The neurobehavioral cognitive status examination: accuracy of the 'Screen-Metric' approach in a clinical sample," Journal of Clinical Psychology, vol. 53, no. 7, pp. 733-737, 1997.

[89] M. A. Hely, W. G. J. Reid, M. A. Adena, G. M. Halliday, and J. G. L. Morris, "The Sydney Multicenter Study of Parkinson's disease: the inevitability of dementia at 20 years," Movement Disorders, vol. 23, no. 6, pp. 837-844, 2008.

[90] G. Guyatt, D. Cook, and B. Haynes, "Evidence based medicine has come a long way," British Medical Journal, vol. 329, no. 7473, pp. 990-991, 2004.

[91] M. E. Morris, C. L. Martin, and M. L. Schenkman, "Striding out with Parkinson disease: evidence-based physical therapy for gait disorders," Physical Therapy, vol. 90, no. 2, pp. 280-288, 2010.

[92] N. L. Ashworth, K. E. Chad, E. L. Harrison, B. A. Reeder, and S. C. Marshall, "Home versus center based physical activity programs in older adults," Cochrane Database of Systematic Reviews, no. 1, Article ID CD004017, 2005.

[93] M. Schenkman, D. Hall, R. Kumar, and W. M. Kohrt, "Endurance exercise training to improve economy of movement of people with Parkinson disease: three case reports," Physical Therapy, vol. 88, no. 1, pp. 63-76, 2008.

[94] M. van Nimwegen, A. D. Speelman, K. Smulders et al., "Design and baseline characteristics of the ParkFit study, a randomized controlled trial evaluating the effectiveness of a multifaceted behavioral program to increase physical activity in Parkinson patients," BMC Neurology, vol. 10, article 70, 2010.

[95] C. J. Liu and N. K. Latham, "Progressive resistance strength training for improving physical function in older adults," Cochrane Database of Systematic Reviews, no. 3, Article ID CD002759, 2009.

[96] D. T. Wade, R. J. E. M. Smeets, and J. A. Verbunt, "Research in rehabilitation medicine: methodological challenges," Journal of Clinical Epidemiology, vol. 63, no. 7, pp. 699-704, 2010. 


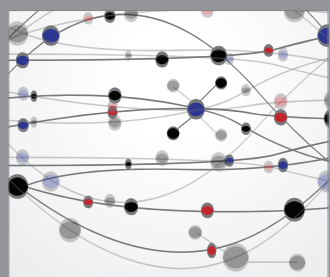

The Scientific World Journal
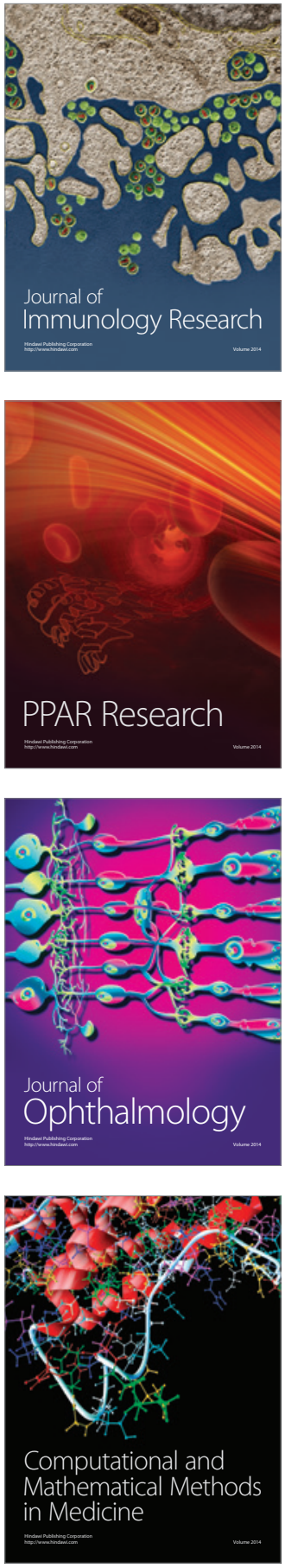

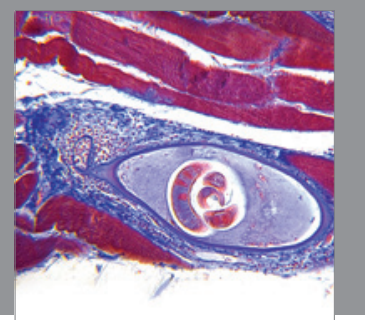

Gastroenterology

Research and Practice
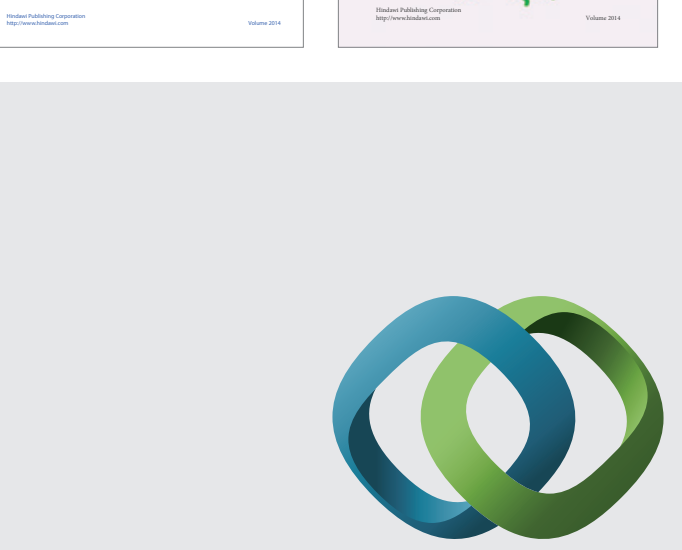

\section{Hindawi}

Submit your manuscripts at

http://www.hindawi.com
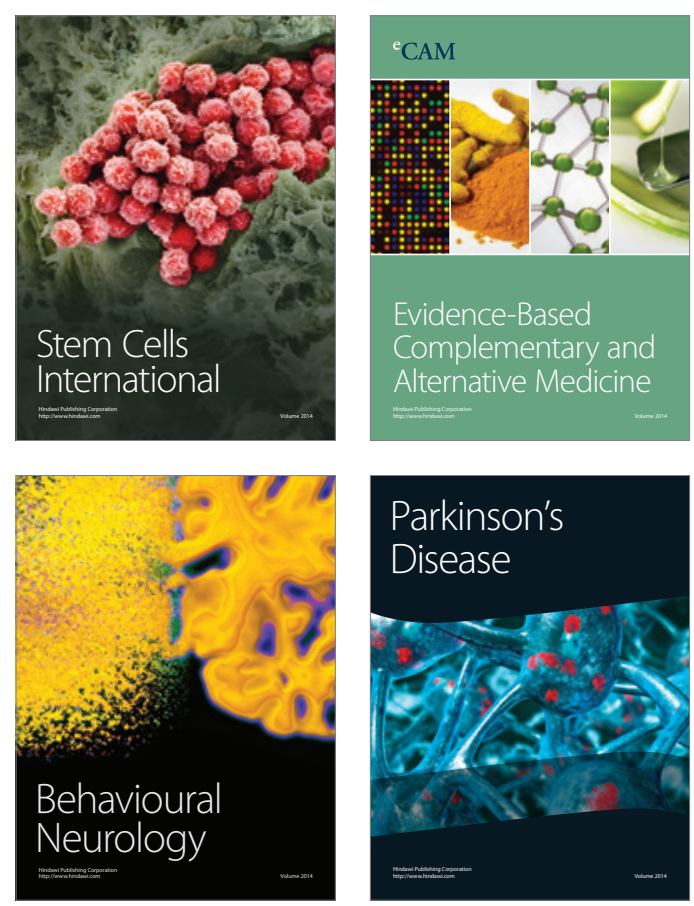

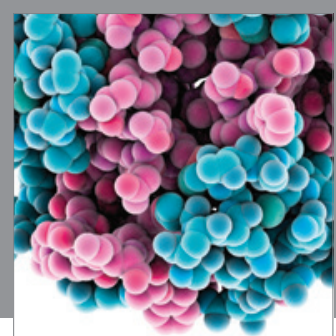

Journal of
Diabetes Research

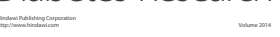

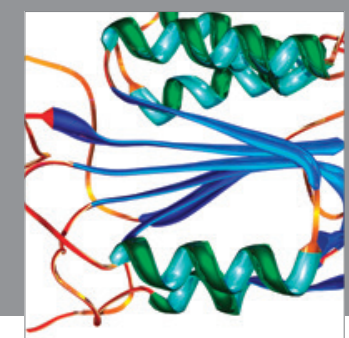

Disease Markers
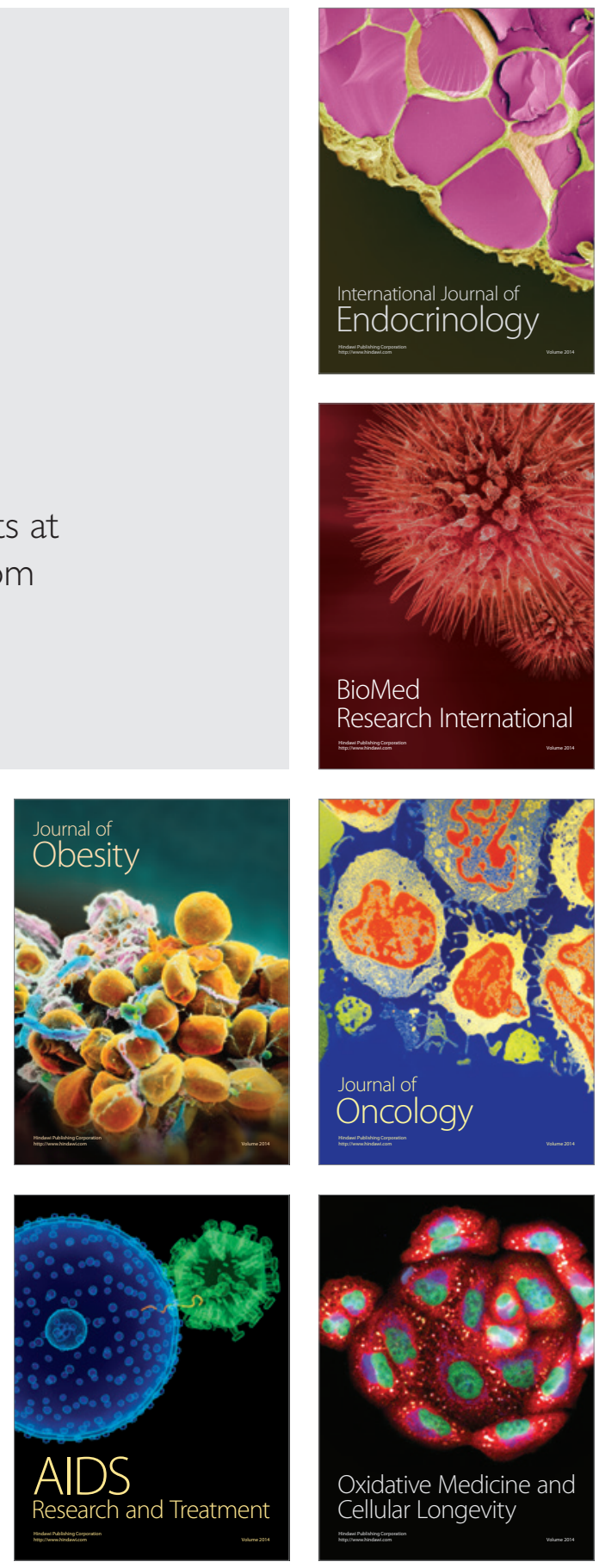